\title{
Continuous monitoring of cerebrovascular pressure reactivity in patients with head injury
}

\author{
Christian Zweifel, M.D., ${ }^{1}$ Andrea Lavinio, M.D., ${ }^{1}$ Luzius A. Steiner, M.D., Ph.D., ${ }^{2}$ \\ Danila Radolovich, M.D., ${ }^{1}$ Peter Smielewski, Ph.D., ${ }^{1}$ Ivan Timofeev, M.R.C.S., ${ }^{1}$ \\ Magdalena Hiler, M.D., ${ }^{1}$ Marcella Balestreri, M.D. ${ }^{1}$ Peter J. KirkPatrick, \\ F.R.C.S.(SN), ${ }^{1}$ John D. Pickard, F.Med.Sci., ${ }^{1}$ Peter Hutchinson, M.D., Ph.D., ${ }^{1}$ \\ and Marek Czosnyka, Ph.D. ${ }^{1}$
}

${ }^{1}$ Academic Neurosurgical Unit, University of Cambridge Clinical School, Cambridge, United Kingdom; and ${ }^{2}$ Department of Anaesthesia, University Hospital Basel, Switzerland

\begin{abstract}
Object. Cerebrovascular pressure reactivity is the ability of cerebral vessels to respond to changes in transmural pressure. A cerebrovascular pressure reactivity index $(\mathrm{PRx})$ can be determined as the moving correlation coefficient between mean intracranial pressure (ICP) and mean arterial blood pressure.

Methods. The authors analyzed a database consisting of 398 patients with head injuries who underwent continuous monitoring of cerebrovascular pressure reactivity. In 298 patients, the PRx was compared with a transcranial Doppler ultrasonography assessment of cerebrovascular autoregulation (the mean index [Mx]), in 17 patients with the PET-assessed static rate of autoregulation, and in 22 patients with the cerebral metabolic rate for $\mathrm{O}_{2}$. Patient outcome was assessed 6 months after injury.

Results. There was a positive and significant association between the PRx and $\mathrm{Mx}\left(\mathrm{R}^{2}=0.36, \mathrm{p}<0.001\right)$ and with the static rate of autoregulation $\left(\mathrm{R}^{2}=0.31, \mathrm{p}=0.02\right)$. A PRx $>0.35$ was associated with a high mortality rate $(>$ $50 \%)$. The PRx showed significant deterioration in refractory intracranial hypertension, was correlated with outcome, and was able to differentiate patients with good outcome, moderate disability, severe disability, and death. The graph of PRx compared with cerebral perfusion pressure (CPP) indicated a $U_{-}$-shaped curve, suggesting that too low and too high CPP was associated with a disturbance in pressure reactivity. Such an optimal CPP was confirmed in individual cases and a greater difference between current and optimal CPP was associated with worse outcome (for patients who, on average, were treated below optimal $\mathrm{CPP}\left[\mathrm{R}^{2}=0.53, \mathrm{p}<0.001\right]$ and for patients whose mean CPP was above optimal CPP $\left.\left[\mathrm{R}^{2}=-0.40, \mathrm{p}<0.05\right]\right)$. Following decompressive craniectomy, pressure reactivity initially worsened (median -0.03 [interquartile range -0.13 to 0.06 ] to 0.14 [interquartile range $0.12-0.22$ ]; $\mathrm{p}<0.01$ ) and improved in the later postoperative course. After therapeutic hypothermia, in $17(70.8 \%)$ of 24 patients in whom rewarming exceeded the brain temperature threshold of $37^{\circ} \mathrm{C}$, ICP remained stable, but the average PRx increased to 0.32 ( $\mathrm{p}<$ 0.0001 ), indicating significant derangement in cerebrovascular reactivity.

Conclusions. The PRx is a secondary index derived from changes in ICP and arterial blood pressure and can be used as a surrogate marker of cerebrovascular impairment. In view of an autoregulation-guided CPP therapy, a continuous determination of a PRx is feasible, but its value has to be evaluated in a prospective controlled trial.
\end{abstract}

(DOI: $10.3171 /$ FOC.2008.25.10.E2)

\section{KeY WoRds - cerebral perfusion pressure • cerebrovascular pressure reactivity $\quad \bullet \quad$ head injury intracranial pressure monitoring $\quad$ - static rate of autoregulation}

$\mathrm{C}$ EREBROVASCULAR pressure reactivity reflects the capability of smooth muscle tone in the walls of in transmural pressure (cerebral vessels constrict in response to an increase in CPP, and vice versa). Cerebro-

\footnotetext{
Abbreviations used in this paper: $\mathrm{ABP}=$ arterial blood pressure; $\mathrm{CBF}=$ cerebral blood flow; $\mathrm{CMRO}_{2}=$ cerebral metabolic rate for $\mathrm{O}_{2}$; $\mathrm{CPP}=$ cerebral perfusion pressure $\mathrm{Mx}=$ mean index of cerebrovascular autoregulation; GCS = Glasgow Coma Scale; PRx = cerebrovascular pressure reactivity index; $\mathrm{SRoR}=$ static rate of autoregulation.
}

vascular pressure reactivity represents a key element of cerebral autoregulation, although the two terms should not be used interchangeably because vascular responses can occur outside the range of cerebral autoregulation. ${ }^{7,25}$ With increasing $\mathrm{ABP}$, intact cerebrovascular pressure reactivity will lead to vasoconstriction and a reduction of cerebral blood volume. Under the condition of a finite pressure-volume compensatory reserve, this reduction of cerebral blood volume will produce a decrease in ICP, a condition that is usually not met in patients after a decompressive craniectomy or in those with an external ventricular drain. When cerebrovascular pressure reac- 


\section{Zweifel et al.}

tivity is impaired, cerebral blood volume-and therefore ICP - will increase or decrease passively (in the same direction) in response to changes in $\mathrm{ABP}$.

The assessment of cerebrovascular pressure reactivity (using the PRx) and its prognostic importance in patients with traumatic head injury have been recognized early, although its measurement remained a challenge without manipulation of $\mathrm{ABP}{ }^{7,24}$ Some authors have suggested that cerebrovascular pressure reactivity could be derived from the characteristic pulse waveform from $\mathrm{ABP},{ }^{26,27}$ although this has never been demonstrated to work in clinical practice. Perhaps changes in ABP are too fast (a fraction of a second) to mobilize an active vasoregulatory response. Slow waves of ABP, lasting from 20 seconds to 3 minutes, are almost always present in patients receiving mechanical ventilation and are of sufficient magnitude to

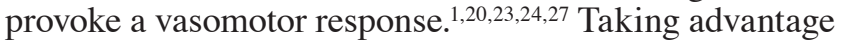
of this fact, cerebrovascular pressure reactivity can be determined continuously without manipulation of ABP by monitoring the response of ICP to such changes in mean ABP.

The introduction of a computer-aided approach to calculate cerebrovascular pressure reactivity and to monitor it continuously was introduced in $1997 .{ }^{8}$ Since then, the use of the PRx in patients with head injury has progressed. A collection of almost 400 cases with continuous monitoring of the PRx has been created, supplemented by intermittent recordings of blood flow velocity in the middle cerebral artery. We intended to review our own findings regarding the PRx and to outline the potential use of this index to facilitate intensive care treatment of patients with severe head injuries.

\section{Methods}

\section{Patient Characteristics and Pressure Reactivity Monitoring}

Our head injury database includes 398 patients with head injuries admitted to the Neuro-Rehabilitation Annex and Neuro Critical Care Unit of Addenbrooke's Hospital, Cambridge, between 1991 and 2007. Several different treatment protocols were used within this period. From 1991 to 1993, the treatment regimen was the so-called "neurorehabilitation annex." Later, a CPP-oriented therapy was administered. ${ }^{18,28}$ Since 2003, a mixed ICP/CPP protocol has been in use with a restricted use of vasopressors. ${ }^{22}$

The database population included 314 men (79\%) and 84 women (21\%), ranging in age from 16 to 79 years old (median age 33 years). The median GCS score at admission was 7 and ranged from 3 to $13 ; 22 \%$ of patients had an initial GCS score $>8$, but their condition deteriorated later, requiring neurocritical care.

Beginning in September 1996, continuous cerebrovascular pressure reactivity monitoring was included in the computer-assisted algorithm. In 2003, an improved version of the bedside software (ICM+; http://www.neurosurg.cam.ac.uk/icmplus) was launched..$^{30}$ In 298 patients the PRx was retrospectively calculated from intermittent computer raw data recordings of ABP and ICP. Continuous monitoring of ICP using Codman intraparenchymal

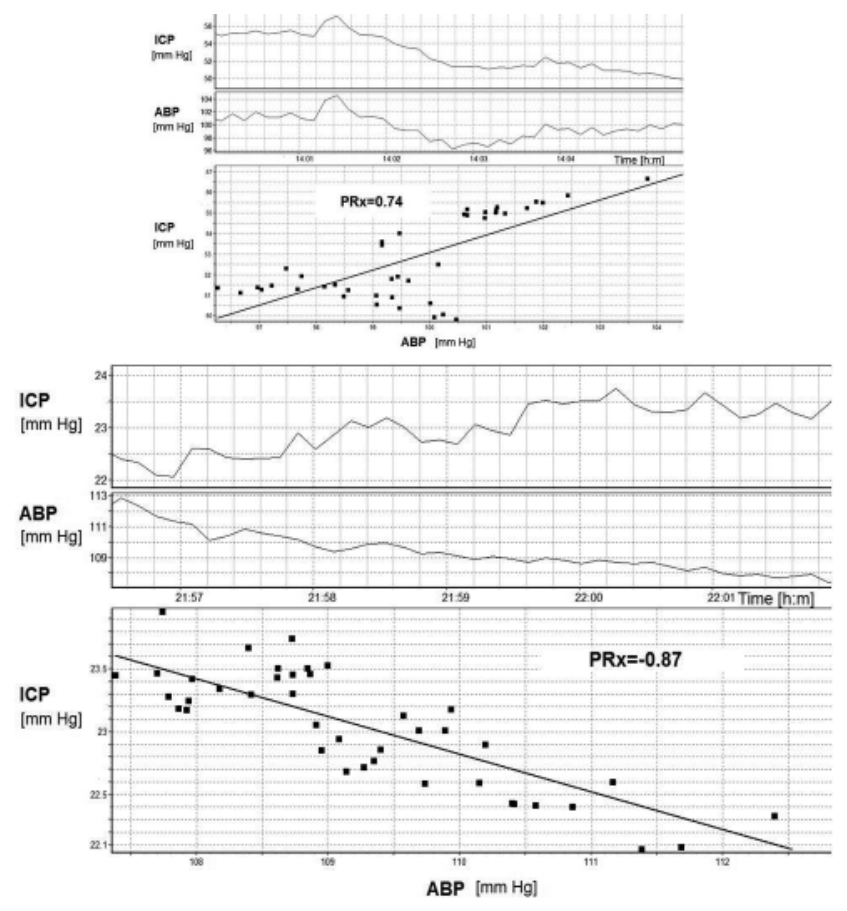

FIG. 1. Graphs showing correlation coefficients between 40 consecutive values (8-second average) of ICP and ABP indicating the state of cerebrovascular pressure reactivity. Upper: A positive PRx correlation (0.74) suggests impaired cerebrovascular pressure reactivity, that is, passive transmission of changes in ABP to ICP. Lower: A negative PRx correlation $(-0.87)$ indicates good pressure reactivity. Any changes in ABP produces inverse changes in ICP.

ICP sensors (in 87\%) or Camino ICP intraparenchymal sensors or external transducers connected to an extraventricular drain (before 1995) was supplemented by other modalities, including direct ABP from the radial artery (in all patients), brain tissue oxygenation (using Neurotrend or Licox, in 74 patients), cortical laser Doppler blood flow (in 31 patients), and PET CBF and $\mathrm{CMRO}_{2}$ (in 39 patients).

\section{Data Analysis}

Using computational methods, the PRx was determined by calculating the correlation coefficient between 40 consecutive, time-averaged data points (8-second periods) of ICP and ABP. A positive PRx signified a positive gradient of the regression line between the slow components of ABP and ICP, which has been shown to be associated with a passive behavior of a nonreactive vascular bed. A negative value of PRx reflected normally reactive cerebral vessels, as ABP waves provoke inversely correlated waves in ICP (Fig. 1).

Similarly, in patients with intermittent transcranial Doppler ultrasonography recordings, the $\mathrm{Mx}$ was calculated as a Pearson correlation coefficient of 40 consecutive samples (320 seconds, or 5 minutes and 20 seconds) of time-averaged (8 seconds) CPP and flow velocity values. The correlation between CPP and middle cerebral artery mean flow velocity values was measured using transcranial Doppler ultrasonography. Flow velocity over 


\section{Continuous monitoring of cerebrovascular pressure reactivity}

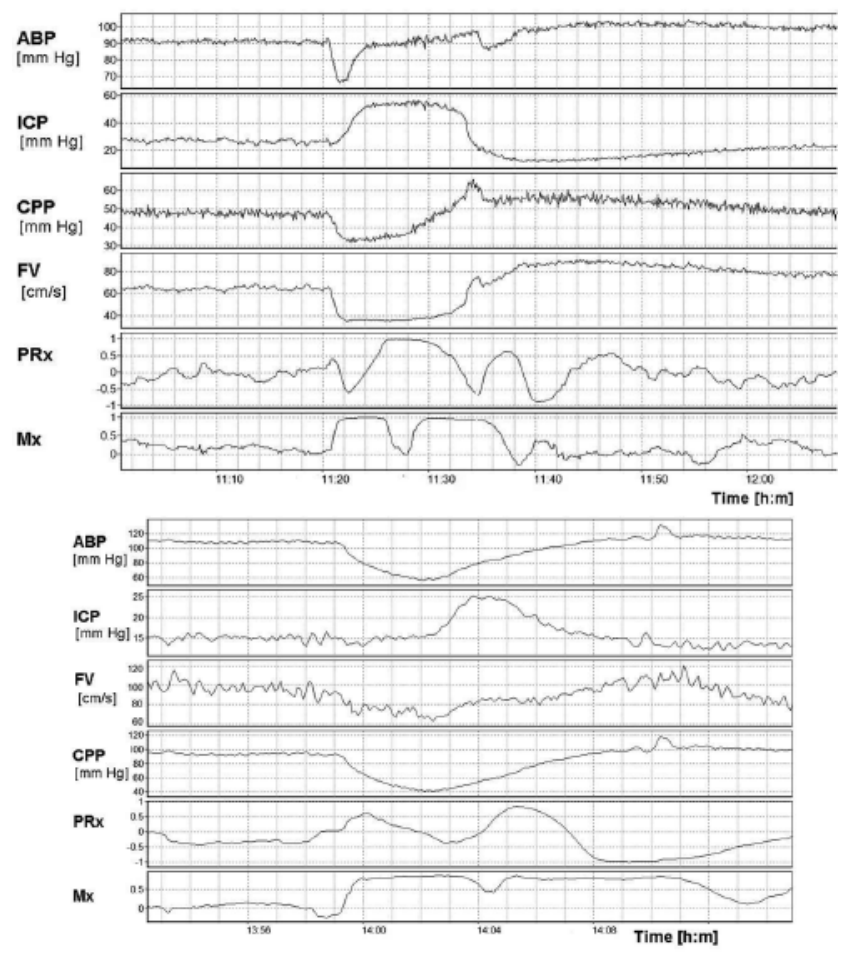

FIG. 2. Examples of Mx (transcranial Doppler-based index of autoregulation) and PRx calculated continuously and changing over time. During plateau waves in ICP, both indices (PRx and Mx) are temporarily assuming values close to +1 , indicating vasodilation (upper). During incidental arterial hypotension, vasodilation is caused by a decrease in CPP (lower). FV = flow velocity.

a limited period can be analyzed using a regression plot of flow velocity (y-axis) versus CPP (x-axis). If points show a linear model, the correlation coefficient is reasonably positive or negative. A positive association between CPP and flow velocity (positive $\mathrm{Mx}$ values) indicates passive dependence of blood flow on CPP, and therefore defective autoregulation. Zero or negative $\mathrm{Mx}$ values implicate active cerebrovascular responses to changes in CPP, and therefore preserved autoregulation.

In both indices (PRx and $\mathrm{Mx}$ ) the time interval over which ICP, ABP, CPP, and flow velocity are averaged (8 seconds in this study) is not very crucial. Any period from 6-10 seconds works in practice. Averaging is used to suppress the influence of the pulse wave and part of the respiratory wave. All slower waves potentially contain information about cerebrovascular pressure reactivity and autoregulation of CBF. The length of the time window (5 minutes) is long enough to contain a period of the longest waves ( 3 minutes) but not too long to be affected by longterm trends in pressure.

In PET CBF studies, SRoR was calculated as the percentage change in cerebrovascular resistance (cerebrovascular resistance $=\mathrm{CBF} / \mathrm{CPP}$ ) divided by the percentage change in CPP; thus, a value of $100 \%$ represents ideal autoregulation and $0 \%$ represents completely nonfunctional autoregulation.

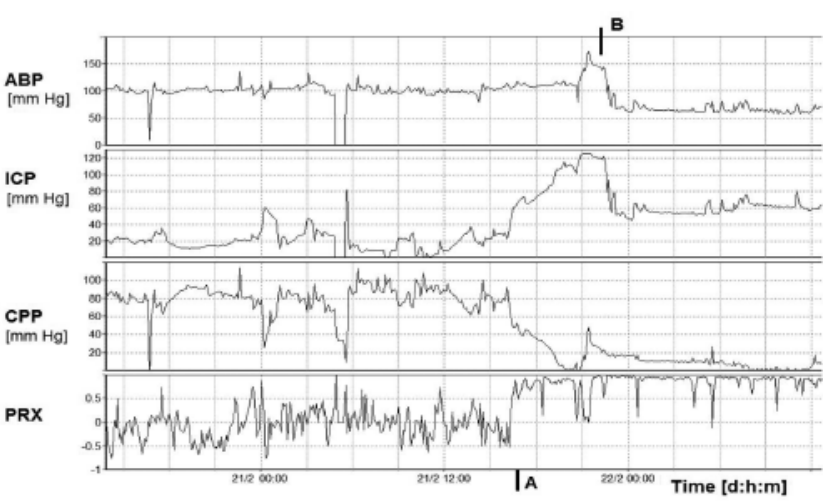

FIG. 3. Example of continuous monitoring of PRx in a patient who died after developing suddenly refractory intracranial hypertension. The value of $P R x$ increased to $>0.5$ past point $A$. Six hours later, brainstem herniation was indicated by drop in ABP (point $B$ ). The interval between the switch of PRx to radically positive values and a drop in CPP below $50 \mathrm{~mm} \mathrm{Hg}$ was 45 minutes.

\section{Results}

Comparison Between the PRx and the Mx

We compared the PRx with the transcranial Doppler-derived Mx in 298 patients with head injuries. The $\mathrm{PRx}$ showed a relatively good correlation with the $\mathrm{Mx}\left(\mathrm{R}^{2}\right.$ $=0.36, \mathrm{p}<0.001) .{ }^{8}$ Both indices presented similar changes during recording intervals (Fig. 2). In an example of episodes of refractory intracranial hypertension, the PRx was capable of detecting temporary or permanent impairment of autoregulation (Fig. 3). 5,10

\section{Comparison of PRx with PET CBF and $\mathrm{CMRO}_{2}$}

The hypothesis that the PRx is an indicator of autoregulation was further validated in PET studies. The PRx was simultaneously compared with the global SRoR measured using PET CBF, which has shown a significant association $\left(R^{2}=0.31, p=0.02 ; 17\right.$ patients), especially for low static values. ${ }^{32}$ After using PET to determine $\mathrm{CMRO}_{2}, \mathrm{CMRO}_{2}$ has been shown to be negatively associated with $\operatorname{PRx}\left(\mathrm{R}^{2}=0.21, \mathrm{p}=0.018 ; 22\right.$ patients). ${ }^{31}$ The correlation between PRx and the $\mathrm{O}_{2}$ extraction fraction was fitted into a quadratic model $\left(\mathrm{R}^{2}=0.55, \mathrm{p}=0.0001\right)$. This model suggests that both low $\mathrm{O}_{2}$ extraction fraction (indicating luxury perfusion, hyperemia, or necrotic tissue) and high $\mathrm{O}_{2}$ extraction fraction (representing poor perfusion or ischemia) are associated with disturbed pressure reactivity.

\section{Comparison of PRx With Intracranial Hypertension, CT, and Outcome}

Abnormal cerebrovascular pressure reactivity is associated with a fatal outcome after head injury. ${ }^{2}$ In a retrospective analysis of 193 patients with head injuries with continuous monitoring of $\mathrm{PRx}$, pressure reactivity was worse in patients who died (22\%) compared with those who survived $(0.19 \pm 0.26$ vs $0.04 \pm 0.18$, respectively; $p$ $<0.0002)$. Mortality modeled as a function of PRx was unevenly distributed (Fig. 4). The PRx values greater than 0.25 indicated a mortality rate of $69 \%$, as opposed to a 

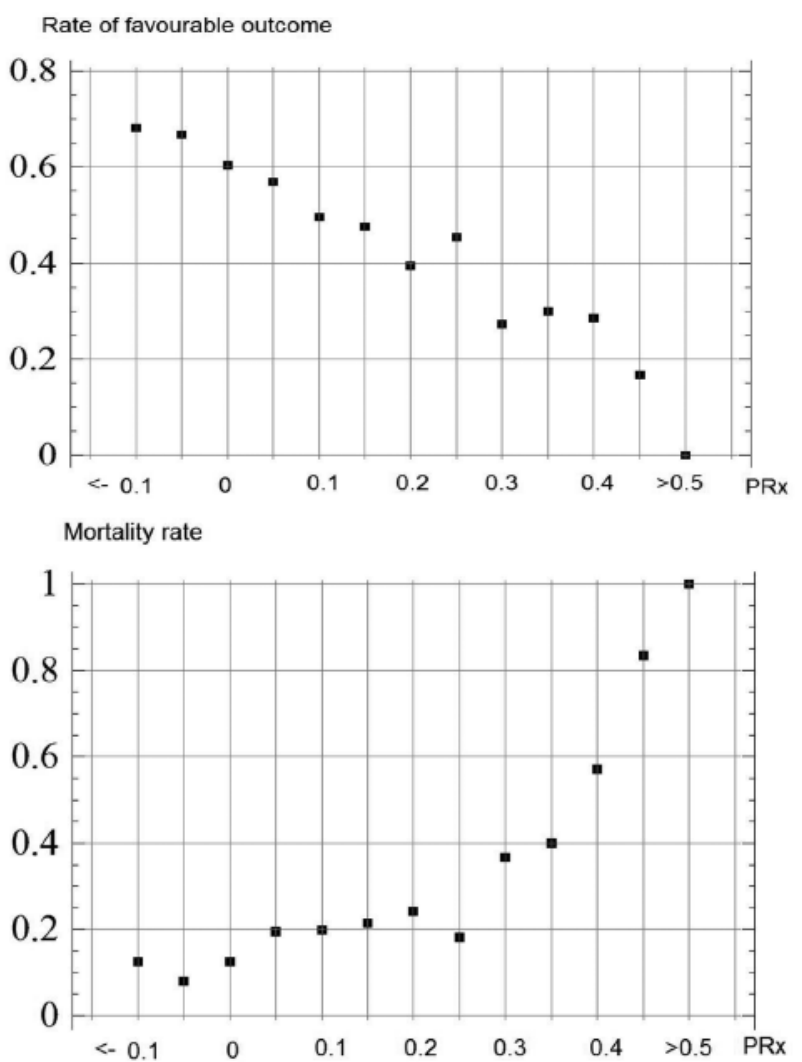

FIG. 4. Graphs of the relationship between the rate of favorable outcome, mortality rate, and PRx in a cohort of 398 patients with head injuries with continuous monitoring (dots represent mean values). Upper: The PRx indicating worse cerebrovascular reactivity (becoming more positive) reduced the rate of favorable outcome uniformly. Lower: The mortality rate increased abruptly to $>50 \%$ when the PRx became radically positive (>0.35).

mortality rate $<20 \%$ in patients with a PRx value $<0.25$ ( $p<0.0001$; chi-square test). These results confirmed those in previous studies, showing that PRx is one of the most important predictors of death after brain trauma. ${ }^{31,33}$ In this updated analysis of 398 patients, outcome showed a close linear relation to PRx, whereas outcome was unevenly distributed with ICP (Fig. 5); this finding supports the assumption of the contribution of PRx to patient outcome.

In a stepwise multivariate analysis, PRx as well as ICP emerged as independent predictors of outcome $\left(\mathrm{R}^{2}=\right.$ $0.37 ; \mathrm{p}<0.0001) .{ }^{5}$ Other independent predictors were age and GCS score, ${ }^{4}$ whereas GCS score was not an independent predictor in a recent patient cohort. ${ }^{3}$

In a study comparing outcome with PRx and the CT classification (according to the Marshall CT classification system), PRx showed a better correlation with outcome $\left(\mathrm{R}^{2}=-0.36, \mathrm{p}<0.0002\right)$ than the $\mathrm{CT}$ classification $\left(\mathrm{R}^{2}=\right.$ $-0.23, p=0.018)$ in a subgroup of 107 patients. ${ }^{13}$ Separating patients into 2 groups (one with positive and the other with negative PRx values) shows that the mortality rate differed considerably (28.6\% in those with positive PRx values vs $9.5 \%$ in those with negative PRx values), even though both groups did not show statistically significant differences in ICP and CPP values and CT scores.
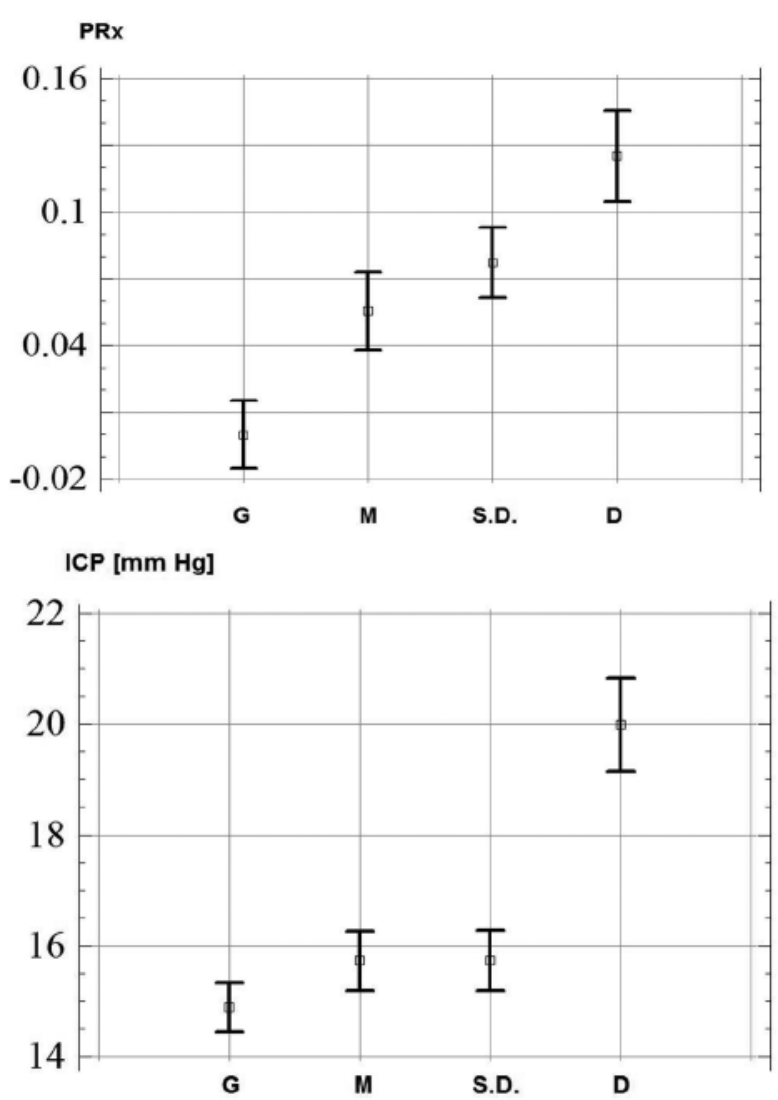

FIG. 5. Distributions of PRx (upper) and mean ICP (lower) in 398 patients with head injuries in different outcome groups $(G=$ good outcome, $M=$ moderate disability, S.D. = severe disability, $D=$ patients who died). Outcome was assessed 6 months after injury. Vertical bars are $95 \%$ confidence intervals. Upper: The PRx values uniformly increased with worse outcome. Lower: Mean ICP is the same in all outcome groups with the exception of patients who died.

\section{Optimal CPP Therapy}

The relationship between cerebrovascular pressure reactivity and CPP shows a U-shaped curve, suggesting that too low or too high CPP values are unsuitable from the point to maintain good cerebrovascular reserve. Figure 6 upper shows PRx (averaged per patient) in a group of 398 patients with continuous PRx monitoring. Both too low (ischemia) and too high CPP (hyperemia and a secondary increase in ICP) are adversarial, hence, CPP should be optimized to maintain CPP in the most favorable state. The question has been asked of whether such optimal CPP (the CPP that assures the best condition for cerebrovascular pressure reactivity) can be identified in individual patients and followed over time. Steiner and colleagues $^{33}$ reported that in two-thirds of 114 patients with head injuries (a subgroup of patients from 19972000), PRx plotted against CPP displayed a U-shaped curve. Consequently, optimal CPP can be evaluated in most individual cases (Fig. 6 lower). In this retrospectively evaluated cohort, patient outcome correlated with the difference between the averaged CPP and optimal CPP for patients who were treated on average below optimal 

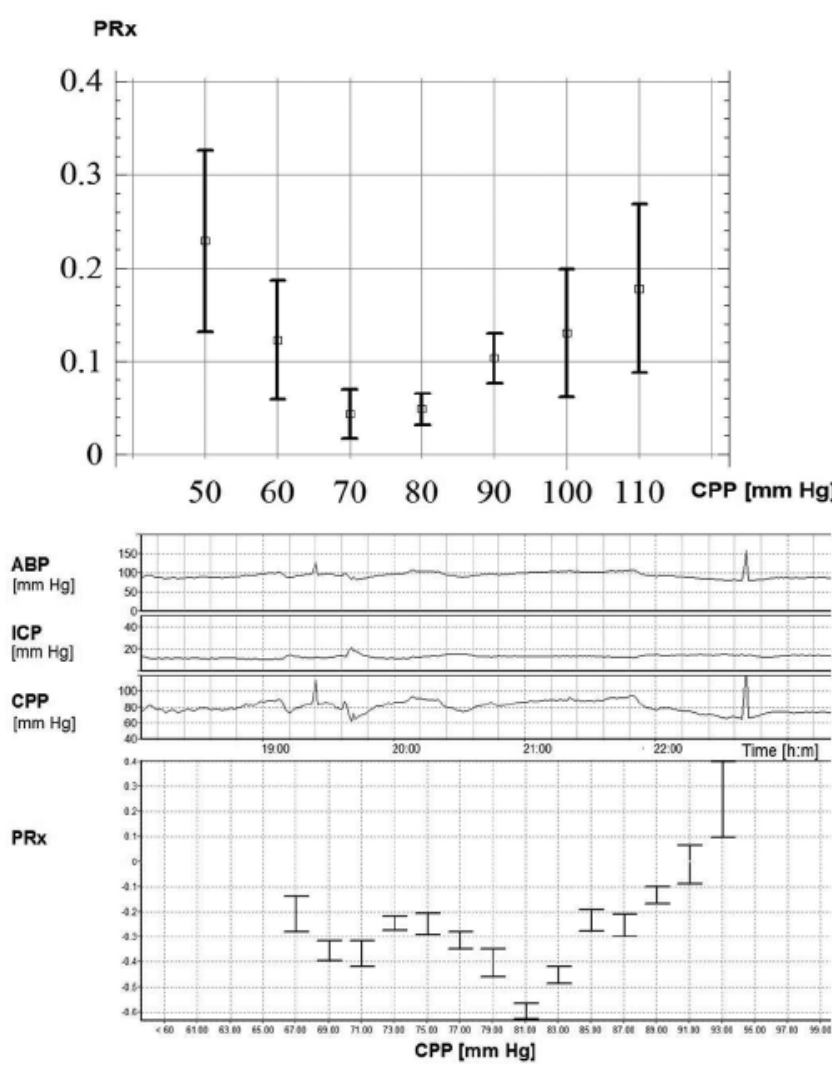

FIG. 6. Graphs of the relationship between the PRx and CPP. Upper: Values of CPP plotted in $10 \mathrm{mmHg}$ intervals for 398 patients monitored continuously. The plot suggests that at too low CPP, vascular reactivity is impaired, which could produce ischemia, and at too high CPP vascular reactivity is also impaired, aggravating the risk of hyperemia. Lower: Results of a statistical analysis of a large cohort of patients can be applied to individual patients. This graph is a 6-hour interval for a patient with potentially stable ICP, ABP, and CPP. The PRx/CPP plot allows one to precisely assess optimal CPP at $81 \mathrm{~mm} \mathrm{Hg}$.

CPP $\left(\mathrm{R}^{2}=0.53, \mathrm{p}<0.001\right)$ and for patients whose mean CPP was above optimal CPP $\left(\mathrm{R}^{2}=-0.40, \mathrm{p}<0.05\right)$.

An algorithm was proposed in which the PRx/CPP relationship was evaluated for 4-6 hours to find optimal CPP and minimize (by altering ABP) the distance between CPP and optimal CPP. ${ }^{33}$ Such adjustments of CPP should not be made too frequently, but hourly corrections may be feasible. This concept needs to be tested in a prospective trial.

\section{Cerebrovascular Pressure Reactivity After Decompressive Craniotomy}

The pressure-volume curve has an exponentially increasing shape, which is particularly steep after head injury. This curve becomes flat after decompressive craniectomy, making a prerequisite assumption for PRx as an index of cerebrovascular reactivity probably invalid. In a retrospective study with 17 patients who underwent decompressive craniectomy, PRx deteriorated postoperatively from a median of -0.03 (interquartile range -0.13 to 0.06 ) to 0.14 (interquartile range $0.12-0.22 ; \mathrm{p}<0.01$ ) initially and improved in the later postoperative course

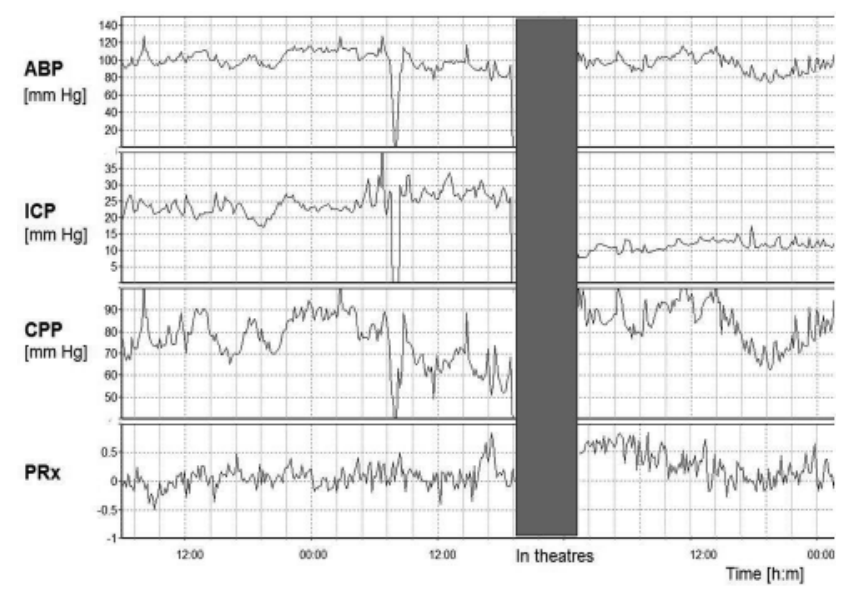

FIG. 7. Graph of a patient's PRx after a craniectomy. The mean ICP level was $>30 \mathrm{~mm} \mathrm{Hg}$ before surgery, with mean PRx $\sim+0.1$. After the craniectomy ICP decreased to $12 \mathrm{~mm} \mathrm{Hg}, \mathrm{CPP}$ improved to $87 \mathrm{mmHg}$, and the PRx increased to 0.45 but later decreased to 0 .

(Fig. 7). ${ }^{35}$ The PRx in patients who did not undergo decompression did not change significantly with time (unless their condition deteriorated).

\section{Cerebrovascular Pressure Reactivity During Hypothermia and Rewarming}

The clinical benefit of hypothermia in the treatment of refractory intracranial hypertension is not clear yet. ${ }^{12}$ In 24 patients with head injuries, PRx was monitored during hypothermia/rewarming. ${ }^{19}$ Hypothermia helped to control increased ICP and did not impair pressure reactivity. Slow rewarming up to $37.0^{\circ} \mathrm{C}$ (rate of rewarming $\left.0.2^{\circ} \mathrm{C} / \mathrm{hr}\right)$ did not significantly increase ICP $(18.6 \pm 6.2$ $\mathrm{mmHg})$ or PRx $(0.06 \pm 0.18)$. However, in $17(70.8 \%)$ of 24 patients who underwent rewarming and whose brain temperature exceeded $37^{\circ} \mathrm{C}$, ICP remained stable, but the average mean PRx increased to $0.32 \pm 0.24$ ( $p<0.0001$ ), indicating a significant derangement in cerebrovascular pressure reactivity (Fig. 8).

\section{Discussion}

Continuous ICP monitoring is an essential modality in neurointensive care, even though no randomized controlled trial has proven an effect of ICP monitoring on overall patient outcome. Intracranial pressure is a complex variable that comprises useful information about cerebrospinal pathophysiology. The association of slow waves of $\mathrm{ABP}$ and ICP has been previously described as an index of the cerebral autoregulatory state. ${ }^{14,23,34}$ Using a computed moving correlation technology to relate ICP to $\mathrm{ABP}, \mathrm{PRx}$ can be determined as a coefficient ranging from -1 (intact pressure reactivity) to +1 (impaired pressure reactivity).

In our patient database, there was a significant correlation of PRx and the transcranial Doppler-derived $\mathrm{Mx}$, which confirmed that PRx was a valid alternative for continuous autoregulation assessment. However, whereas $\mathrm{Mx}$ assesses vascular responses of an individual branch of a basal artery, PRx reflects a global vasomotor reactiv- 


\section{Zweifel et al.}

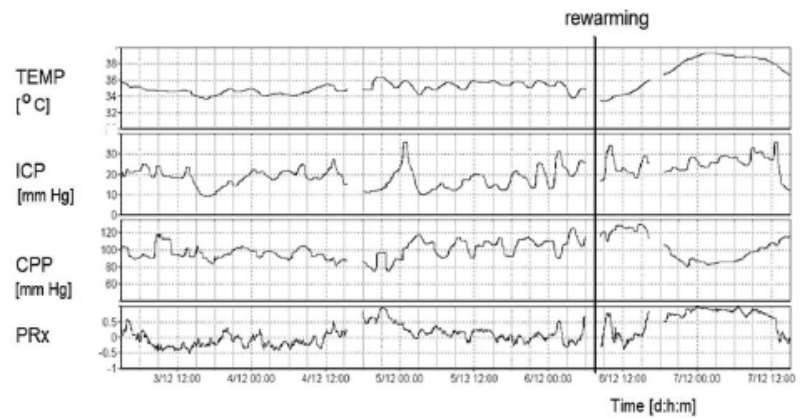

FIG. 8. Graph of PRx changing during rewarming after the period of hypothermia. When brain temperature increased to $>37.5^{\circ} \mathrm{C}, \mathrm{PRx}$ increased to values indicative of deranged cerebrovascular pressure reactivity, with only a slight increase in ICP (to $25 \mathrm{~mm} \mathrm{Hg}$ ).

ity reserve. After head injury, the brain vascular bed may have some of the relevant capacity in a heterogeneous distribution preserved, ${ }^{9,6,11}$ and therefore PRx is a useful indicator of a trend toward an improving or deteriorating global cerebrovascular dilatory reserve. In addition, continuous (24-hour) autoregulation monitoring using $\mathrm{Mx}$ is technically very difficult because the present technology requires a trained person at the patient's bedside to adjust the Doppler probes during monitoring. This technical difficulty makes Mx impractical for continuous long-term monitoring. The PRx, on the other hand, is not restricted by such technical limitations.

Impaired cerebrovascular pressure reactivity was also associated with reduction of $\mathrm{CMRO}_{2}$ and $\mathrm{CBF}$, which emphasizes the validity of the PRx as a surrogate marker of cerebrovascular impairment. Although PRx and Mx showed a good correlation in our database, the validation of a correlation between Mx and SRoR (PET) in a study by Steiner et al. ${ }^{32}$ was unsuccessful. This finding could suggest that PRx is a more robust estimator of autoregulation than Mx.

Cerebrovascular pressure reactivity correlates well with outcome after brain trauma. Whereas ICP only differentiates patients with fatal outcome from those who survive at 6 months, PRx distinguished between patients with good outcome, moderate disability, severe disability, and death. The correlation of PRx with patient outcome is independent of mean ICP: in a stepwise multivariate analysis both variables were included in the model independently. Moreover, patients with head injuries who are treated close to the optimal CPP-as determined by the PRx calculation - had a better outcome in a retrospectively evaluated cohort. This data emphasizes the potential benefits of including autoregulation data in treatment guidelines, as previously noted by several other authors. ${ }^{17,21,29}$ Based on a study with more than 120 patients, Howells et al. ${ }^{15}$ suggested that an ICP-oriented therapy should be used in patients whose PRx is $>0.13$, whereas below that threshold, a CPP-oriented therapy is more beneficial for outcome. This combined approach using 2 different treatment protocols has been shown to produce a better overall outcome in their retrospective analysis, but might be difficult to implement in a prospective treatment concept because pressure reactivity can vary rapidly (Fig. 2). Nev- ertheless, a real-time updated treatment protocol including PRx in a CPP-oriented therapy can easily be included in a standard neurointensive care unit, and requires only minimal changes in technology. Steiner et al. ${ }^{33}$ proposed a strategy concerning how to approach optimal CPP in clinical practice. A PRx-guided CPP therapy is one possible autoregulation-oriented approach, although PRx does not precisely represent autoregulation. But the fact that there is a time-dependent effect of PRx on outcome suggests that PRx is a useful alternative on which an autoregulation-oriented therapy could be based.

Decompressive craniectomy leads to reduction in ICP and adequate CPP levels can be achieved at lower mean ABP levels. ${ }^{35}$ Decompressive craniectomy has also been shown to improve cerebral oxygenation and microdialysis values, although the effect on neurological outcome has yet to be proven. ${ }^{14,16}$ Initial worsening of PRx after decompressive craniectomy should be confirmed in other studies and is not yet fully understood, ${ }^{36}$ but outcome was significantly correlated with postoperative PRx values. ${ }^{35,36}$

Using cerebrovascular pressure reactivity as a surrogate marker could also be very useful under particular intensive care conditions such as hypothermia. The results of our cohort have emphasized the importance of management of systemic temperature after rewarming. Although an average PRx changing from 0.06 to 0.32 might appear to represent a rather unimpressive increase, as we show in Fig. 4, the mortality rate increases steeply at a PRx value $>0.3$.

Prospective data in the literature on a pressure reactivity-guided intensive care therapy are missing, which is also the major limitation of our data given that all analyses were performed retrospectively. The concept of pressure reactivity-guided intensive care therapy has to be proven in a prospective randomized trial. A further limitation of this study is the use of a very specific, although standard, intensive care management strategy, which limits the transfer of our results to centers that use distinctly different management strategies.

\section{Conclusions}

The PRx is a secondary index calculated using ICP and APB and can be used as a surrogate marker of cerebrovascular impairment. Continuous long-term monitoring of PRx allows the determination of the CPP at which cerebrovascular pressure reactivity reaches its optimal value in individual patients. The benefit of such an autoregulation-oriented approach should be evaluated in a prospective study.

\section{Disclosure}

Funding for this study was provided by a grant from the Medical Research Council (No. G9439390, ID 65883) to J.D.P., M.C., P.S., P.J.K., and P.H. Peter Hutchinson, M.D., Ph.D., is supported by a Health Foundation Senior Scientist Fellowship from the Academy of Medical Sciences. Marek Czosnyka, Ph.D. is on leave from Warsaw University of Technology, Poland. The software for brain monitoring ICM+ (www.neurosurg.cam.ac.uk/icmplus) is licensed by the University of Cambridge (Cambridge Enterprise). Peter Smielewski, 


\section{Continuous monitoring of cerebrovascular pressure reactivity}

Ph.D., and Marek Czosnyka, Ph.D., have a financial interest in a part of the licensing fee.

\section{Acknowledgments}

We thank all our colleagues who participated in the head injury monitoring program between 1991 and 2007 in Cambridge, United Kingdom, whose experience, knowledge, and clinical material helped us to write this paper: Mrs. D. Chatfield, Mrs. P. Al-Rawi, Mrs. C. Turner, Dr. S.K. Piechnik, Mr. P.C. Whetfield, Mr. P. Minhas, Dr. M. Soehle, Mr. R. Kett-White, Dr. E. Schmidt, Dr. B. Schmidt, Mr. A. Kumar, Prof. A. Raabe, Dr. M. Schuhmann, Mr. E.Guazzo, Dr. B.F. Matta, Prof. A. Gupta, Professor D.K. Menon, and the nursing and research staff from the Neuro Critical Care Unit and Wolfson Brain Imaging Centre.

\section{References}

1. Aaslid R, Lindegaard KF, Sorteberg W, Nornes H: Cerebral autoregulation dynamics in humans. Stroke 20:45-52, 1989

2. Balestreri M, Czosnyka M, Steiner LA, Hiler M, Schmidt EA, Matta B, et al: Association between outcome, cerebral pressure reactivity and slow ICP waves following head injury. Acta Neurochir Suppl 95:25-28, 2005

3. Balestreri M, Czosnyka M, Chatfield DA, Steiner LA, Schmidt EA, Smielewski P, et al: Predictive value of Glasgow Coma Scale after brain trauma: change in trend over the past ten years. J Neurol Neurosurg Psychiatry 75:161-162, 2004

4. Balestreri M, Czosnyka M, Hutchinson P, Steiner LA, Hiler M, Smielewski P, et al: Impact of intracranial pressure and cerebral perfusion pressure on severe disability and mortality after head injury. Neurocrit Care 4:8-13, 2006

5. Balestreri M, Czosnyka M, Steiner LA, Schmidt E, Smielewski P, Matta B, et al: Intracranial hypertension: what additional information can be derived from ICP waveform after head injury? Acta Neurochir (Wien) 146:131-141, 2004

6. Bouma GJ, Muizelaar JP, Choi SC, Newlon PG, Young HF: Cerebral circulation and metabolism after severe traumatic brain injury: The elusive role of ischemia. J Neurosurg 75:685-693, 1991

7. Cold GE, Jensen FT: Cerebral autoregulation in unconscious patients with brain injury. Acta Anaesthesiol Scand 22:270280, 1978

8. Czosnyka M, Smielewski P, Kirkpatrick P, Laing RJ, Menon D, Pickard JD: Continuous assessment of the cerebral vasomotor reactivity in head injury. Neurosurgery 41:11-19, 1997

9. Czosnyka M, Smielewski P, Kirkpatrick P, Menon DK, Pickard JD: Monitoring of cerebral autoregulation in head-injured patients. Stroke 27:1829-1834, 1996

10. Czosnyka M, Smielewski P, Piechnik S, Schmidt EA, Al-Rawi PG, Kirkpatrick PJ, et al: Hemodynamic characterization of intracranial pressure plateau waves in head-injury patients. J Neurosurg 91:11-19, 1999

11. Enevoldsen EM, Jensen FT: Autoregulation and CO2 responses of cerebral blood flow in patients with acute severe head injury. J Neurosurg 48:689-703, 1978

12. Henderson WR, Dhingra VK, Chittock DR, Fenwick JC, Ronco JJ: Hypothermia in the management of traumatic brain injury. A systematic review and meta-analysis. Intensive Care Med 29:1637-1644, 2003

13. Hiler M, Czosnyka M, Hutchinson P, Balestreri M, Smielewski P, Matta B, et al: Predictive value of initial computerized tomography scan, intracranial pressure, and state of autoregulation in patients with traumatic brain injury. J Neurosurg 104:731-737, 2006

14. Ho CL, Wang CM, Lee KK, Ng I, Ang BT: Cerebral oxygenation, vascular reactivity, and neurochemistry following decompressive craniectomy for severe traumatic brain injury. J Neurosurg 108:943-949, 2008
15. Howells T, Elf K, Jones PA, Ronne-Engström E, Piper I, Nilsson $\mathrm{P}$, et al: Pressure reactivity as a guide in the treatment of cerebral perfusion pressure in patients with brain trauma. $\mathbf{J}$ Neurosurg 102:311-317, 2005

16. Hutchinson PJ, Corteen E, Czosnyka M, Mendelow AD, Menon DK, Mitchell P, et al: Decompressive craniectomy in traumatic brain injury: the randomized multicenter RESCUEicp study (www.RESCUEicp.com). Acta Neurochir Suppl 96:17-20, 2006

17. Lang EW, Chesnut RM: A bedside method for investigating the integrity and critical thresholds of cerebral pressure autoregulation in severe traumatic brain injury patients. $\mathbf{B r} \mathbf{J}$ Neurosurg 14:117-126, 2000

18. Lang EW, Chesnut RM: Intracranial pressure and cerebral perfusion pressure pressure after severe head injury. New Horiz 3:400-409, 1995

19. Lavinio A, Timofeev I, Nortje J, Outtrim J, Smielewski P, Gupta A, et al: Cerebrovascular reactivity during hypothermia and rewarming. Br J Anaesth 99:237-244, 2007

20. Lundberg N: Continuous recording and control of ventricular fluid pressure in neurosurgical practice. Acta Psychiatr Neurol Scand Suppl 36:1-193, 1960

21. Mascia L, Andrews PJ, McKeating EG, Souter MJ, Merrick MV, Piper IR: Cerebral blood flow and metabolism in severe brain injury: the role of pressure autoregulation during cerebral perfusion pressure management. Intensive Care Med 26:202-205, 2000

22. Menon DK, Wheeler DW, Wilkins IA, Phillips PD, Fletcher SJ, Penfold NW, et al: Integrated approaches to academic anaesthesia-the Cambridge experience. Anaesthesia 59:785-792, 2004

23. Muizelaar JP, Ward JD, Marmarou A, Newlon PG, Wachi A: Cerebral blood flow and metabolism in severely head-injured children. Part 2: autoregulation. J Neurosurg 71:72-76, 1989

24. Overgaard J, Tweed WA: Cerebral circulation after head injury. 1. Cerebral blood flow and its regulation after closed head injury with emphasis on clinical correlations. J Neurosurg 41:531-541, 1974

25. Paulson OB, Strandgaard S, Edvinsson L: Cerebral autoregulation. Cerebrovasc Brain Metab Rev 2:161-192, 1990

26. Portnoy HD, Chopp M, Branch C, Shannon M: Cerebrospinal fluid pulse waveform as an indicator of cerebral autoregulation. J Neurosurg 56:666-678, 1982

27. Piper IR, Miller JD, Dearden NM, Leggate JR, Robertson I: System analysis of cerebrovascular pressure transmission: an observational study in head injured patients. J Neurosurg 73:871-880, 1990

28. Rosner MJ, Rosner SD, Johnson AH: Cerebral perfusion pressure: management protocol and clinical results. J Neurosurg 83:949-962, 1995

29. Sahuquillo J, Poca MA, Ausina A, Báguena M, Gracia RM, Rubio E: Arterio-jugular differences of oxygen (AVDO2) for bedside assessment of $\mathrm{CO} 2$-reactivity and autoregulation in the acute phase of severe head injury. Acta Neurochir (Wien) 138:435-444, 1996

30. Smielewski P, Czosnyka M, Steiner L, Belestri M, Piechnik S, Pickard JD: ICM+: software for on-line analysis of bedside monitoring data after severe head trauma. Acta Neurochir Suppl 95:43-49, 2005

31. Steiner LA, Coles JP, Czosnyka M, Minhas PS, Fryer TD, Aigbirhio FI, et al: Cerebrovascular pressure reactivity is related to global cerebral oxygen metabolism after head injury. J Neurol Neurosurg Psychiatry 74:765-770, 2003

32. Steiner LA, Coles JP, Johnston AJ, Chatfield DA, Smielewski P, Fryer TD, et al: Assessment of cerebrovascular autoregulation in head-injured patients. A validation study. Stroke 34:2404-2409, 2003

33. Steiner LA, Czosnyka M, Piechnik SK, Smielewski P, Chat- 


\section{Zweifel et al.}

field D, Menon, DK, et al: Continuous monitoring of cerebrovascular pressure reactivity allows determination of optimal cerebral perfusion pressure in patients with traumatic brain injury. Crit Care Med 30:733-738, 2002

34. Steinmeier R, Bauhuf C, Hubner U, Bauer RD, Fahlbusch R, Laumer R, et al: Slow rhythmic oscillations of blood pressure, intracranial pressure, microcirculation, and cerebral oxygenation. Dynamic interrelation and time course in humans. Stroke 27:2236-2243, 1996

35. Timofeev I, Czosnyka M, Nortje J, Smielewski P, Kirkpatrick P, Gupta A, et al: Effect of decompressive craniectomy on intracranial pressure and cerebrospinal compensation following traumatic brain injury. J Neurosurg 108:66-73, 2008
36. Wang EC, Ang BT, Wong J, Lim J, Ng I: Characterization of cerebrovascular reactivity after craniectomy for acute brain injury. Br J Neurosurg 20:24-30, 2006

37. Wilson JT, Pettigrew LE, Teasdale GM: Structured interviews for the Glasgow Outcome Scale and the extended Glasgow Outcome Scale: guidelines for their use. J Neurotrauma 15:573-585, 1998

Manuscript submitted June 10, 2008.

Accepted August 6, 2008.

Address correspondence to: Marek Czosnyka, Ph.D., Academic Neurosurgery, Box 167, Addenbrooke's Hospital, Cambridge CB2 2QQ, United Kingdom. email: MC141@medschl.cam.ac.uk. 